is the most valuable for comparative purposes. For instance, on the sample represented by the curve marked "Gelatin," it will be observed that the range of temperature between the points of liquefaction of the I per cent and the 2 per cent mixture is about one degree; also, that between 4 per cent and 6 per cent concentration it is about one degree, while between 2 per cent and 3 per cent the temperature range is about $18^{\circ}$. While in this particular instance the $10^{\circ}$ point is no more valuable than the $16^{\circ}$ poitit for a standard jelly-value temperature, yet taking this curve in conjunction with the others illustrated herewith, it will be seen that $10^{\circ} \mathrm{C}$. is a good average working temperature-point for observation of the entire line of glues and gelatins.

RESEARCH ILABORATORY

JOHNSON \& JOHNSON

NEW BruNswick, N. J.

\section{A NEW METHOD FOR THE QUANTITATIVE ESTIMATION OF VAPORS IN GASES}

\section{A DIFFERENTIAL PRESSURE METHOD}

By Harold $S$. Davis and Maky Dayidson Davis

Received March 27, 1918

\section{INTRODUCTION}

In a former paper a new form of tensimeter was described for measuring the partial pressure, in a mixture of inert gases, from any liquid or from its solution in a nonvolatile substance.

The ease with which these experiments could be carried out led us to experiment with modified forms of this apparatus, having in view the development of a method for the estimation of small quantities of vapors in inert gas mixtures. ${ }^{2}$

\section{THEORY OF METHOD}

According to the principle often referred to as Dalton's Law of Partial Pressures, the vapor pressure from a liquid is independent of the kind of gas above it, provided the gas is inert. Deviations from this law are well known, but it holds with surprising accuracy in the case of a mixture of benzene and air at atmospheric pressure, as has already been shown by one of us. ${ }^{1}$

Consider two closed flasks connected, as in Fig. I, by a manometer and filled with air at atmospheric pressure. If now a small sealed bulb containing a volatile liquid be broken in each, the liquid will partially evaporate, and if the temperatures of the flasks remain the same, the same additional pressure will be developed in each, so that the manometer connecting them will register no difference in pressure. Even if the temperatures of the flasks do vary, no difference in pressure will be recorded until there is a relative difference in temperature between them.

Now suppose that one of the flasks had contained a certain quantity of the vapor of the volatile liquid corresponding to a pressure less than the saturation

I "The Extraction of Aromatic Hydrocarbons from Gases by Means of Liquid Absorbents," Harold S. Davis, University of Manitoba Publications, February, 1917.

2 Most of the work was done during the early part of the summer of 1917 and the results under the same heading as that of this paper were given to the Canadian Advisory Council and to the Imperial Munitions Board of Canada. United States Patent No, 1,272,922 on this method was issued July 16,1918 . pressure. When the small bulb of liquid was broken in this one, the liquid would not add all its vapor pressure to the pressure already in the flask, for part of that was already due to its vapor. It. would add. only the amount of pressure necessary to bring its pressure up to saturation; and since the total saturation pressure was added to the pure air in the other flask, the manometer connecting the two would register a pressure equal to the pressure of vapor in the original gas.

Two important points should be noted here:

I-The partial pressure of any particular vapor in a sample of gas is independent of the temperature of the gas, provided that the total pressure on the gas remains constant while the volume can change with the temperature, and provided the vapor remains always unsaturated and obeys the simple gas laws.

2-The difference in pressure developed between the two flasks, one of which contains air and vapor, and the other air free from vapor, will vary as the absolute temperature, provided the relative temperatures of the flasks remain the same; that is, for practical purposes, the difference in the pressure is independent of variations of temperature.

An apparatus constructed on this principle will, therefore, measure a definite quantity, viz., the pressure of the vapor in a gas at any particular gas pressure.

The differential pressure which develops between the two flasks is equal to the original partial pressure of the vapor in the gas, when the total pressure on the gas is equal to the atmospheric pressure at the time of the experiment. This can be reduced to standard conditions in the following way:

Let $P$ be the atmospheric pressure at the time of the experiment.

Let $\mathrm{P}$ 。 be normal atmospheric pressure $=76 \mathrm{~cm}$. of mercury.

Let $\mathrm{X}$ be the differential pressure developed between the flasks.

Then $\frac{X P_{0}}{P}$ is equal to the partial pressure of the vapor in the gas when the total pressure of the gas is $P_{0}$. As was pointed out before, the value $\frac{X P_{0}}{P}$ is independent of the temperature provided every component of the gas remains unsaturated.

In a similar way the total of the partial pressures of two or more vapors may be reduced to its value for a total standard pressture on the gas.

However, though this partial pressure of a vapor is independent of the temperature, the actual weight of the vapor contained in unit volume of the gas depends on the temperature. For one vapor this may be calculated from the partial pressure on the assumption that the vapor gives the same partial pressure as it would if it were a true gas at that temperature and molecular concentration.

\section{SOURCES OF ERROR}

I-The permanent gases in one of the flasks may dissolve in the liquid to a greater extent than those in the other flask and thus lower the pressure on that side. 
2-The dissolved gases may lower the vapor pressure of the liquid to a greater extent in one flask than in the other and thus decrease the additional pressure developed in the former.

3-If there are other vapors present, these will partially dissolve in the liquid in the bottom of the flask, and thus their own partial pressure in the gas will be less, while, in addition, the vapor pressure from the liquid will be lowered.

If the gases in the two flasks are the same, these errors are negligible, for the only error is caused by the gas in one flask being at a slightly higher pressure than the gas in the other so that more of it will dissolve in the liquid. If, however, the gases are not the same, only careful experimentation will show the magnitude of the errors.

It is not necessary that the substance in the sealed glass bulbs should be in the liquid state, for, in the case of a substance which can exist as a solid under the conditions of the experiment, it is of course possible to estimate the amount of its vapor present in the gas by the same method. We have actually estimated quantities of benzene in gases at temperatures lower than $5^{\circ}$, using bulbs filled with frozen benzene. This will be described in another paper. A solution which has a vapor pressure of the particular substance to be estimated greater than that actually in the gas could be used under certain conditions.

\section{EXPERIMENTAL PART}

The apparatus used is of the form shown in Fig. I. Into the ground glass stopper of each of the flasks tubes are sealed; one of these terminates about I cm.

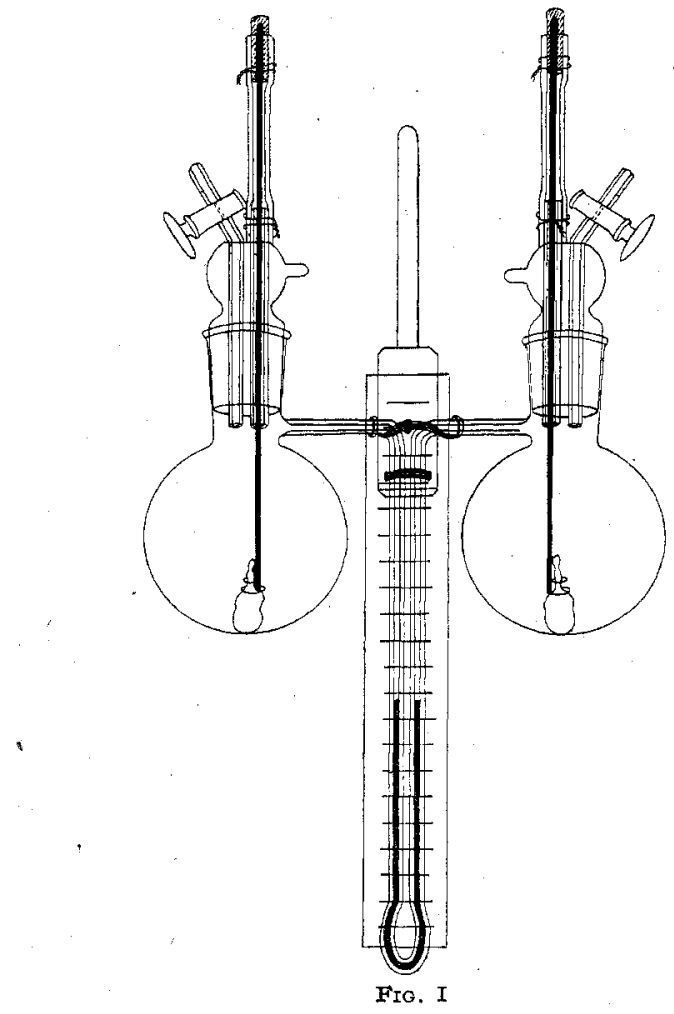

above the stopper and through it a metal rod passes to the bottom of the flask, projecting above the stopper to a distance of $10 \mathrm{~cm}$. The upper end of this rod is screwed into a short piece of metal rod of larger diameter. Over this rod, and extending down over the tubing which projects above the stopper, is slipped a piece of tightly fitting, rubber pressure tubing. The rubber joints are made tight by means of vacuum grease and wire. The second tube passing through the stopper is closed at the upper end by a stopcock. It simply provides a means for the passage of gases into or out of the flask and can as well be sealed into the side of the neck of the flask or the upper part of the manometer tube. In later work we have used it sealed into the manometer. To the bottom of the metal rod a small glass bulb is attached by means of small soldered supports and fine copper wire. This bulb, which has a thin bottom, contains sealed up inside some of the liquid whose vapor is to be estimated. By pushing down the rod at the proper time, or giving it a slight tap, the bulb at the end can be broken, thus liberating the liquid it contains. The rubber tubing then draws the rod back into place. The left flask is filled with the gas to be examined, at a pressure slightly above atmospheric. When the temperatures of the gases in the two flasks become the same, their pressures are equalized to that of the atmos phere by opening the stopcocks, which are then closed. This step is necessary if the temperature of the room or thermostat in which the experiment is being done varies to any extent.

Next, the small sealed bulbs containing the liquid are broken by pushing on the rods and the apparatus is allowed to stand, with an occasional shaking, until the manometer levels cease changing. The time required to reach equilibrium is $I_{0}$ to $15 \mathrm{~min}$. and 30 to $60 \mathrm{~min}$. for flasks of $\mathrm{I}_{40} \mathrm{cc}$. and $340 \mathrm{cc}$. capacity, respectively. In routine work it is possible to hasten the evaporation of the liquid by heating the flasks, either gently with a flame or by immersion in a warm bath. In such a case it is necessary to guard against a leak through the extra pressure developed.

'Two corrections must be applied to the difference in the mercury levels to obtain the true difference in pressure:

$\mathrm{I}-$ Reduction of the mercury height to $0^{\circ} \mathrm{C}$. This can usually be neglected.

2-Correction for the increase in volume in the right-hand flask and the decrease in volume in the left-hand one, dute to the movement of the liquid in the manometer tube. If, however, the flasks are fairly large and the bore of the manometer tube small, this correction can also be generally neglected.

Unfortunately it was impossible, at first, to obtain satisfactory apparatus and much of the following experimental work had to be done with flasks closed by corks instead of glass stoppers. Rubber stoppers cannot be used as they rapidly absorb benzene vapor and so cause serious errors. If the corks are boiled in soft paraffin they will hold a vacuum fairly well but are much inferior to glass stoppers. A little soft paraffin on the stoppers makes gas-tight joints, and to hold them securely in place thick rubber bands are slipped over their tops and fixed to small catches on the necks of the flasks. 
In the first part of this work we introduced the gases into the flasks by simple displacement, but it is surprising how much gas is needed to completely displace the air from even a small flask and most of our troubles arose from overlooking this fact. About 5 liters will displace nearly all the air from a $300 \mathrm{cc}$. flask. We have since devised a slightly modified form of apparatus (Fig. II) which is filled by first evacuating the flask and then attaching to the gas supply. As will be seen, in this form the two flasks are close together, and the manometer tube is led out in front from them, so that the flasks may be placed in a bath of any kind while the manometer $\mathrm{rem}$ ain $\mathrm{s}$ outside. Further, there is a stopcock above the mercury in the manometer tube on the side nearest the flask to be filled with the gas to be analyzed. This stopcock is closed before evacuating the flask. It allows the FIG. II-STDE VIEW MODIFIED FORM OF whole apparatus to be (Only One Flask Shown)

inverted without losing the mercury in the manometer tube.

The evacuation is carried out by a water pump which rapidly reduces the pressure to less than $I \mathrm{~cm}$. of mercury. The residual pressure is measured by a manometer in series with the pump. With this form of apparatus only a small quantity of gas need be collected for analysis. It can be kept, until required, in a small gas holder from which it is displaced into the apparatus by mercury. We have used gas holders of $300 \mathrm{cc}$. capacity with stopcocks at both ends. When one experiment is completed, the apparatus can easily and rapidly be cleaned for the next by drawing hot air through it.

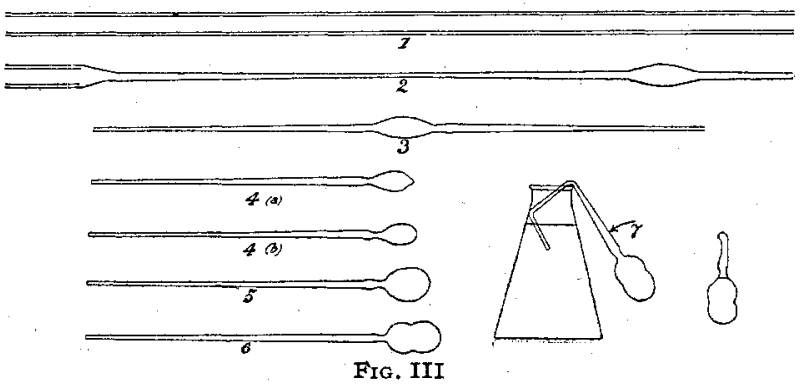

Naturally, in order to avoid fracture, the determination flasks should be of good uniform thickness, especially near the bottom where the little bulbs are broken. We have also lessened the danger of breakage by placing a thin piece of lead in the bottom of each flask. These precautions are useful when the bulb contains a solid, but when dealing with a liquid one need never break the bottom of a flask if the little sealed bulbs containing the liquid are made as shown in Fig. III.

The bulb is blown from a piece of glass tubing, preferably of a diameter not less than $0.8 \mathrm{~cm}$. Our experience has been that if smaller tubing is used, bubbles form in the glass when it is blown; this may, however, be due to incorrect methods of procedure. This tubing, $I$, is first drawn out as in 2 and a slight constriction made on each side, 3 , for convenience later in tying the bulb to the bottom of the rod (see Fig. I). From 4 the round bulb 5 is blown and at once its bottom is heated in a mild flame and blown out in the form 6 . Bulbs of this type will stand a wonderful amount of usage but can be broken on the bottom by a gentle pressure against any surface.

For filling, the neck of the bulb is bent in the form 7 and immersed in a narrow-mouthed vessel full of the desired liquid. On heating gently at intervals, the air is soon driven out, some liquid drawn over, vaporized, and the whole bulb filled with liquid. The liquid is now driven out from the capillary and the bulb full of liquid is sealed off with a small pointed flame at the point 7 . In the case of benzene and toluene we have used a small naked flame for heating, and protected the flask by wrapping with asbestos paper. In this way a bulb can be filled in a few moments. Occasionally, one explodes and takes fire without serious results. If desired, one can work behind a glass screen. In the case of more volatile substances a liquid bath for heating and other such precautions could be used.

Another satisfactory method for filling the bulbs is as follows: The vessel containing the desired liquid together with a number of bulbs immersed in it, as shown in 7 , are placed in a closed glass dish with a removable top, such as a desiccator. Next the air pressure in the desiccator is lowered by pumping out the air and is again brought to atmospheric by opening a stopcock. If this process is repeated a few times the bulbs will be completely filled with liquid.

We have given this description in detail, but when once mastered the manipulation is easy and two persons with only moderate experience in glassblowing can easily prepare twenty complete bulbs filled and sealed in an hour, casualties not being counted.

At first, we performed all the experiments in a thermostat with glass sides, the temperature of which remained constant to a few hundredths of a degree; but this is unnecessary. We have found it quite easy to obtain accurate results in a room where the temperature varied over several degrees.

Several samples of benzene obtained from wellknown firms were used; on being distilled they boiled over constant to less than a tenth of a degree. Many of the preliminary results are omitted.

We attempted to check the benzene content in samples of air, containing known quantities of benzene, made up in the following way: Measured volumes of air, saturated with benzene vapor at a known temperature, were drawn from a $500 \mathrm{cc}$. flask and diluted with air over mercury. In the first 5 experiments given below, a I $40 \mathrm{cc}$. determination flask was filled 
by displacement of air with about $500 \mathrm{cc}$. of the sample of air and benzene vapor. This was a quantity. far from sufficient to displace all the original air, and the results are low.

In Expt. 6, the flask was filled by displacement of mercury and the result is much better. In No. 7 , a bulb containing a known weight of benzene was broken in a. 5 liter flask slightly evacuated. Air was then admitted and the whole shaken up well by means of a small quantity of mercury in the bottom of the flask. After this, a 340 cc. determination flask was filled by displacement of air with the 5 liters of air and benzene vapor which were driven over by displacement with water.

\section{PRELIMTNARY RESULTS}

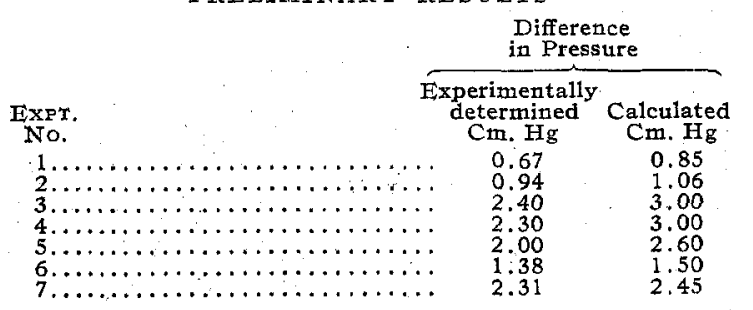

In the final series of determinations a weighed quantity of benzene, less than that required for satura-

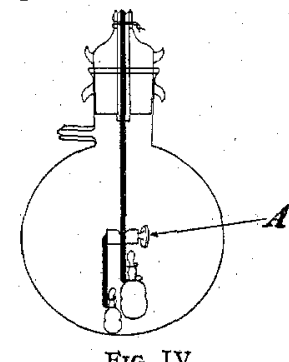

FIG, IV tion,. was introduced directly into the determination flask. This was accomplished by means of a second small, sealed bulb (Fig. IV) which contained a weighed amount of benzene. Its position could be regulated by means, of the attachment screw A, so that on pushing on the rod the small bulb broke, leaving

be broken later. the larger one above it intact, to

In this way the actual pressure developed by the weighed amaunt of benzene could be measured, and the pressure could be checked by breaking the large bulbs according to the method already described.

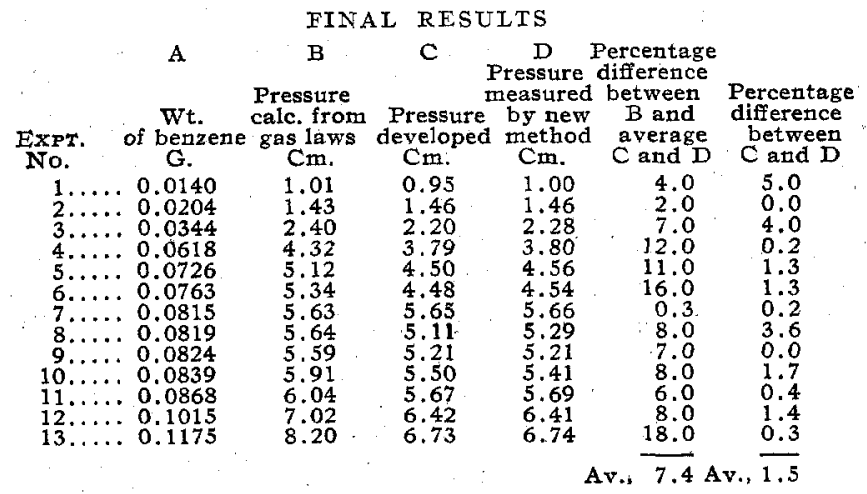

DISCUSSION OF RESULTS

The agreement between the pressures of benzene actually developed and those determined by the new method is satisfactory. The average deviation is I. 5 per cent, but better agreement could undoubtedly have been secured by working in a thermostat, as the temperature of the room varied considerably.

The differences between the pressures actually developed and those calculated from the weights of ben- zene, the volume of the flasks being known, are fairly large; mean deviation, 7 per cent. In each case the pressure developed was less than the calculated.

These deviations may be attributed to two causes: I-Impurities in the benzene and impurities collected from the interior surface of the flask. These impurities when dissolved in the last traces of benzene might lower its vapor pressure until it would cease to evaporate, being in equilibrium with the pressure in the flask. Naturally, this tendency would increase as the amount of benzine pressure in the flask increased.

2-Divergence of the benzene vapor from the simple gas laws caused perhaps by polymerization, but the results show that this effect is not very large for pressures approaching saturation.

\section{SUMMARY}

I-A differential pressure method for the quantitative estimation of vapors in gases has been described.

II-Experimental results are given of the trial of this method for the estimation of quantities of benzene vapor in air.

DEPARTMENT OF CHEMISTRY, UNIVERSITY OF MANITOBA WINAIPEG, CANADA

\section{THE APPLICATION OF THE DIFFERENTIAL PRESSURE METHOD TO THE ESTIMATION OF THE BENZENE AND THE TOTAL LIGHT OIL CONTENT OF GASES}

By Harold S. Davis, Mary Davidson Davis and Donald G. MACG REGOR

Received March 27, 1918

INTRODUCTION

It is unnecessary to dwell on the importance of the aromatic hydrocarbons, particularly benzene and toluene, at the present time, and on the necessity of increasing their output in every possible way.

A great need has been felt, by those engaged in the commercial production of these substances, for methods of analysis requiring only small samples of gas and giving a rapid estimation of the content of these vapors either, collectively or individually. Such methods would make it possible to find the conditions of production necessary to obtain the maximum concentration of each aromatic substance and would also permit the efficiency of absorption processes to be tested at every point.

A method widely used at present for the estimation of these vapors requires the following steps:

I-Their absorption from a large measured quantity of the gas by means of a suitable solvent.

2-The distilling and fractionating of the solution thus obtained according to a definite scheme of operation. ${ }^{1}$

The absorption process most widely adopted for works purposes is the passage of the gas through, a train of wash bottles filled with absorbing oils."

The technique of this process as widely used in the United States has been fully described by F. W. Sperr. ${ }^{3}$

1 H. G. Colman, J. Gas Lighting, 129 (1915), 314-315; H. W. James, J. Soc. Chem. Ind., 85 (1916), 236.

2 R. Lessing, J, Soc Chem. Ind., 36 (1917), 103.

$8 M$ el. and Chem. Eng., 17 (1917), 548, 586, 642. 